\title{
Numerical experiments for multiscale problems in linear elasticity
}

\author{
Orane Jecker and Assyr Abdulle
}

\begin{abstract}
This paper gives numerical experiments for the Finite Element Heterogeneous Multiscale Method applied to problems in linear elasticity, which has been analyzed in [A. Abdulle, Math. Models Methods Appl. Sci. 16, 2006]. The main results for the FE-HMM a priori errors are stated and their sharpness are verified though numerical experiments.
\end{abstract}

\section{Introduction}

Consider the linear elasticity equation in a bounded domain $\Omega \subset \mathbb{R}^{d}$ with a Lipschitz continuous boundary $\partial \Omega$,

$$
\begin{aligned}
-\frac{\partial}{\partial x_{j}}\left(a_{i j k l}^{\varepsilon} \frac{\partial u_{k}^{\varepsilon}}{\partial x_{l}}\right) & =f_{i}, \text { in } \Omega, \\
u_{i}^{\varepsilon} & =0, \text { on } \partial \Omega,
\end{aligned}
$$

for $i=1, \ldots, d$ and where $f \in L^{2}(\Omega)^{d}$. Further assume that $a^{\varepsilon}(x)$ is a fourthorder tensor indexed by $\varepsilon$ describing the microscopic scale of the problem. We define $|M|=(M: M)^{1 / 2}=\left(\sum_{i, j=1}^{d} M_{i j}^{2}\right)^{1 / 2}$ for any square matrix $M$. The tensor is such that $a_{i j k l}^{\varepsilon}(x) \in L^{\infty}(\Omega)$, for all $i, j, k, l=1, \ldots, d$, and

$$
\begin{aligned}
a_{i j k l}^{\varepsilon} & =a_{j i k l}^{\varepsilon}=a_{k l i j}^{\varepsilon}, \\
\alpha|M|^{2} & \leq a^{\varepsilon} M: M, \text { for any symmetric matrix } M, \\
\left|a^{\varepsilon} M\right| & \leq \beta|M|, \text { for any symmetric matrix } M,
\end{aligned}
$$

where $0<\alpha \leq \beta<\infty$. We define the linearized strain tensor $e$, for $i, j=$ $1, \ldots, d$, by

$$
e\left(u^{\varepsilon}\right)=\left(e_{i j}\left(u^{\varepsilon}\right)\right)_{1 \leq i, j \leq d}, \quad e_{i j}\left(u^{\varepsilon}\right)=\frac{1}{2}\left(\frac{\partial u_{i}^{\varepsilon}}{\partial x_{j}}+\frac{\partial u_{j}^{\varepsilon}}{\partial x_{i}}\right) .
$$

The weak formulation of problem (1) reads: find $u^{\varepsilon} \in H_{0}^{1}(\Omega)^{d}$ such that

$$
B\left(u^{\varepsilon}, v\right):=\int_{\Omega} a^{\varepsilon} e\left(u^{\varepsilon}\right): e(v) \mathrm{d} x=\int_{\Omega} f v \mathrm{~d} x=: F(v),
$$

O. Jecker $\cdot$ A. Abdulle

École Polytechnique Fédérale de Lausanne, ANMC, CH-1015 Lausanne

e-mail: orane.jecker@epfl.ch; assyr.abdulle@epfl.ch 
for all $v \in H_{0}^{1}(\Omega)^{d}$. Problem (5) is well-posed thanks to the first Korn inequality, that is

$$
\|v\|_{\mathrm{H}^{1}(\Omega)} \leq C\left(\int_{\Omega}|e(v)|^{2} \mathrm{~d} x\right)^{1 / 2} .
$$

Solving (5) with standard FEM requires the mesh size to be smaller than the fine scale, which is prohibitive if $\varepsilon$ is small. However, the effective dynamics of the problem can be described using homogenization theory $[9,6]$. Using the theory of $H$-convergence $[8,5]$, it can be established that a subsequence of the family of solutions $\left\{u^{\varepsilon}\right\}$ converges weakly to an effective solution $u^{0}$, satisfying the homogenized formulation

$$
B_{0}\left(u^{0}, v\right):=\int_{\Omega} a^{0} e\left(u^{0}\right): e(v) \mathrm{d} x=F(v), \quad \forall v \in H_{0}^{1}(\Omega)^{d} .
$$

The homogenized tensor $a^{0}$ verifies the properties (2)-(4) for some constants $0<\alpha_{0} \leq \beta_{0}<\infty$. Under additional information on the small scale of the tensor, such as periodicity

(H1) $a^{\varepsilon}(x)=a(x / \varepsilon)=a(y)$ is $Y$-periodic in $y$, where $Y=(0,1)^{d}$, explicit equations are available to compute the homogenized tensor $a^{0}$

$$
a_{i j k l}^{0}=\frac{1}{|Y|} \int_{Y} a_{i j k l}(y)+\sum_{h, m=1}^{d} a_{i j h m}(y) \frac{\partial \chi_{h}^{k l}(y)}{\partial y_{m}} \mathrm{~d} y .
$$

The functions $\chi_{h}^{k l} \in W_{\text {per }}(Y)$ are solutions of the micro problems

$$
-\frac{\partial}{\partial y_{j}}\left(a_{i j h m} \frac{\partial \chi_{h}^{k l}}{\partial y_{m}}\right)=\frac{\partial a_{i j k l}}{\partial y_{j}}, \text { in } Y, \text { for } i=1, \ldots, d,
$$

with periodic boundary conditions. The space $W_{\text {per }}(Y)$ is defined as

$$
W_{\text {per }}(Y)=\left\{v \in H_{p e r}^{1}(Y)^{d} \mid \int_{Y} v_{i} \mathrm{~d} y=0, i=1, \ldots, d\right\} .
$$

Remark. Problem (1) can be easily adapted to non-homogeneous Dirichlet and Neumann boundary conditions. A lifting of the Dirichlet data should be considered and extra terms are added to the weak formulations (5) and (6).

In Section 2, we state the FE-HMM method for linear elasticity [1] and in Section 3 we recall the a priori error estimates derived in $[1,2]$. Finally, in Section 4, we illustrate the sharpness of the convergence rates with numerical examples.

\section{Finite Element Heterogeneous Multiscale Method for linear elasticity}

The FE-HMM gives us a macroscopic solution based on a macro to micro modeling without knowing the homogenized tensor $a^{0}$. 
Macro problem. Let $\mathcal{T}_{H}$ be a mesh over $\Omega$ with mesh size $H>>\varepsilon$ given by $H=\max _{K \in \mathcal{T}_{H}} h_{K}$. In each macro element $K$, we consider integration nodes $x_{j, K}$ and weights $\omega_{j, K}$, for $j=1, \ldots, J$, and construct sampling domains $K_{\delta_{j}}=x_{j, K}+\delta[-1 / 2,1 / 2]^{d}$. We define a macro FE space of degree $p$ by

$$
V^{p}\left(\Omega, \mathcal{T}_{H}\right)=\left\{v^{H} \in H_{0}^{1}(\Omega)^{d}\left|v^{H}\right|_{K} \in \mathcal{R}^{p}(K), \quad \forall K \in \mathcal{T}_{H}\right\},
$$

where $\mathcal{R}^{p}(K)$ is the space $\mathcal{P}^{p}(K)$ of polynomials on $K$ of degree at most $p$ if $K$ is a triangle, or the space $\mathcal{Q}^{p}(K)$ of polynomials on $K$ of degree at most $p$ in each variables if $K$ is a rectangle. We construct a macro bilinear form

$$
B_{H}\left(v^{H}, w^{H}\right):=\sum_{K \in \mathcal{T}_{H}} \sum_{j=1}^{J} \frac{\omega_{j, K}}{\left|K_{\delta_{j}}\right|} \int_{K_{\delta_{j}}} a^{\varepsilon}(x) e\left(v_{j}^{h}\right): e\left(w_{j}^{h}\right) \mathrm{d} x,
$$

where $v_{j}^{h}\left(\right.$ resp. $\left.w_{j}^{h}\right)$ is the solution of the micro problem (9) on the sampling domain $K_{\delta_{j}}$. The FE-HMM solution $u^{H}$ verifies

$$
B_{H}\left(u^{H}, v^{H}\right)=F\left(v^{H}\right), \quad \forall v^{H} \in V^{p}\left(\Omega, \mathcal{T}_{H}\right) .
$$

Micro problem. Let $\mathcal{T}_{h}$ be a micro partition over $K_{\delta_{j}}$, for $j=1, \ldots, J$, of mesh size $h<<\varepsilon$, with $h=\max _{K \in \mathcal{T}_{h}} h_{K}$. For each $K_{\delta_{j}}$, we define a micro FE space of degree $q$ as

$$
S^{q}\left(K_{\delta_{j}}, \mathcal{T}_{h}\right)=\left\{v^{h} \in W\left(K_{\delta_{j}}\right)\left|v^{h}\right|_{K} \in \mathcal{R}^{q}(K)^{d}, \quad \forall K \in \mathcal{T}_{h}\right\} .
$$

The micro problems read: find $u_{j}^{h}$ such that $\left(u_{j}^{h}-u_{\text {lin,j }}^{H}\right) \in S^{q}\left(K_{\delta_{j}}, \mathcal{T}_{h}\right)$ and

$$
\int_{K_{\delta_{j}}} a^{\varepsilon}(x) e\left(u_{j}^{h}\right): e\left(v_{j}^{h}\right) \mathrm{d} x=0, \quad \forall v_{j}^{h} \in S^{q}\left(K_{\delta_{j}}, \mathcal{T}_{h}\right),
$$

where $u_{l i n, j}^{H}(x)=u^{H}\left(x_{j, K}\right)+\left(x-x_{j, K}\right) e\left(u_{j}^{h}\right)$ is a linearization of $u^{H}$ taken at the quadrature node $x_{j, K}$. The space $W\left(K_{\delta_{j}}\right)$ sets the coupling between the micro and macro solvers and depends on the choice of boundary conditions in problem (9),

$$
\begin{aligned}
& W\left(K_{\delta_{j}}\right)=W_{\text {per }}\left(K_{\delta_{j}}\right) \text { for periodic coupling, or } \\
& W\left(K_{\delta_{j}}\right)=W_{\text {dir }}\left(K_{\delta_{j}}\right)=H_{0}^{1}\left(K_{\delta_{j}}\right)^{d} \text { for Dirichlet coupling. }
\end{aligned}
$$

\section{A priori error estimates}

In this section we give a priori error estimates for the FE-HMM method, details can be found in $[1,2]$. The error is decomposed into the macro, modeling, and micro error,

$$
\left\|u^{0}-u^{H}\right\| \leq e_{\mathrm{MAC}}+e_{\mathrm{MOD}}+e_{\mathrm{MIC}} .
$$

We assume that the micro solution $\chi^{l m}$ (solution of equation (7)) are smooth enough, i.e., 
(H2)

$$
\begin{aligned}
& \varepsilon \chi^{l m} \in H^{q+1}\left(K_{\delta_{j}}\right)^{d} \text { with }\left\|D^{\alpha}\left(\varepsilon \chi^{l m}\right)\right\|_{L^{\infty}\left(K_{\delta_{j}}\right)} \leq C \varepsilon^{-|\alpha|+1}, \text { for } \alpha \leq q+1 \\
& \text { and } l, m=1, \ldots, d .
\end{aligned}
$$

Theorem 1 ([1]). Let $u^{0}$ and $u^{H}$ be solutions of (6) and (8), respectively. Assume that $u^{0} \in H^{r+1}(\Omega)^{d}$, for some $r>0$, and that the hypothesis (H2) holds. Then,

$$
\begin{aligned}
& \left\|u^{0}-u^{H}\right\|_{\mathrm{H}^{1}(\Omega)} \leq C\left(H^{s}+\left(\frac{h}{\varepsilon}\right)^{2 q}+e_{M O D}\right), \\
& \left\|u^{0}-u^{H}\right\|_{\mathrm{L}^{2}(\Omega)} \leq C\left(H^{s+1}+\left(\frac{h}{\varepsilon}\right)^{2 q}+e_{M O D}\right), \quad s=\min (r, p) .
\end{aligned}
$$

If in addition, the hypothesis (H1) holds, the modeling error is given by

$$
\begin{aligned}
& e_{M O D}=0, \text { for periodic coupling with } \delta / \varepsilon \in \mathbb{N}^{*}, \\
& e_{M O D}=\frac{\varepsilon}{\delta}, \text { for Dirichlet coupling with } \delta>\varepsilon
\end{aligned}
$$

The homogeneous tensor can be approximated during the assembling process of the FE-HMM. For general tensors and sampling domains, we have, in each macro element $K \in \mathcal{T}_{H}$,

$$
a_{i j k l}^{0, h}\left(x_{m, K}\right)=\frac{1}{\left|K_{\delta_{m}}\right|} \int_{K_{\delta_{m}}} a^{\varepsilon}(x) e\left(\varphi_{m, i, j}^{h}\right): e\left(\varphi_{m, k, l}^{h}\right) \mathrm{d} x
$$

where $x_{m, K}$ is a quadrature point in $K$, and $K_{\delta_{m}}$ is the sampling domain around $x_{m, K}$. The functions $\varphi_{m, i, j}^{h} \in W\left(K_{\delta_{m}}\right)$ are solutions of (9) for $i, j \in$ $\{1, \ldots, d\}$. Then, note that if (H1) holds, the tensors $a^{0}$ and $a^{0, h}$ are constants in $\Omega$. The error introduced by computing $a^{0}$ is given by the following Lemma.

Lemma 2. Assume that (H1) holds and that periodic coupling is used with $\delta / \varepsilon \in \mathbb{N}^{*}$. Let $a^{0, h}=\left(a_{i j k l}^{0, h}\right)$ be defined in (10). It holds

$$
\left|a_{i j k l}^{0}-a_{i j k l}^{0, h}\right| \leq C\left(\frac{h}{\varepsilon}\right)^{2 q}
$$

Proof. Follows from [1] (see also $[3,4]$ ).

\section{Numerical Experiments}

In this section we present numerical examples to verify the sharpness of the bounds obtained in Theorem 1 and Lemma 2. In Table 1, we show the best refinement strategies for the optimal $H^{1}$ and $L^{2}$ convergence rates with minimal computational cost. 


\begin{tabular}{|c|c|c|c|c|}
\hline Macro FE & $u^{0}$ & Micro FE & $H^{1}$ norm & $L^{2}$ norm \\
\hline \multirow{2}{*}{$P^{1}$} & \multirow{2}{*}{$H^{2}(\Omega)$} & $P^{1}$ & $\sqrt{N_{m a c}}=N_{m i c}$ & $=N_{m i c}$ \\
\hline & & $P^{2}$ & $=N_{m i c}$ & $\sqrt{N_{m a c}}=N_{m i c}$ \\
\hline \multirow{2}{*}{$P^{2}$} & \multirow{2}{*}{$H^{3}(\Omega)$} & $P^{1}$ & $=N_{m i c}$ & $N_{m a c}^{3 / 2}=N_{m i c}$ \\
\hline & & $P^{2}$ & $\sqrt{N_{m a c}}=N_{m i c}$ & $N_{m a c}^{3 / 4}=N_{m i c}$ \\
\hline
\end{tabular}

Table 1. Best refinement strategies for optimal convergence rates.

We start by showing that the macro convergence rates in $H$ are sharp. Let $\varepsilon=1 / 10$, and consider equation (1) in $\Omega=[0,1]^{2}$ with homogeneous Dirichlet boundary condition, a right-hand side $f=1$, and a tensor $a^{\varepsilon}(x)=a(x / \varepsilon)=$ $a(y)$ given by

$$
a(y)=\left(\begin{array}{ccc}
\sin \left(2 \pi y_{1}\right)+2 & 0 & 0 \\
0 & \sin \left(2 \pi y_{2}\right)+2 & 0 \\
0 & 0 & 10
\end{array}\right), \quad a^{0}=\left(\begin{array}{ccc}
\sqrt{3} & 0 & 0 \\
0 & \sqrt{3} & 0 \\
0 & 0 & 10
\end{array}\right) .
$$

A reference solution for $u^{0}$ is computed on a very fine mesh. We use periodic coupling with $\delta=\varepsilon$ in order to have zero modeling error. Further, the micro degrees of freedom is chosen such that the micro error can be neglected, and take $H=1 / 8,1 / 16,1 / 32,1 / 64,1 / 128$. In Figure $1(\mathrm{a})$, we monitor the $H^{1}$ and $L^{2}$ errors to $u^{0}$ for the piecewise macro and micro FE-HMM. The solution $u^{0}$ is in $H^{2}(\Omega)$ and one can see the linear and quadratic rates for the piecewise $H^{1}$ and $L^{2}$ errors, respectively. However, as one can see in Figure 1(b), $u^{0}$ is not smooth enough to observe the $H^{2}$ and $H^{3}$ convergence rates for the quadratic $H^{1}$ and $L^{2}$ norms, respectively. The optimal rates can be seen in Figure 1(c) where we restrict the errors to a subdomain $\omega \subset \Omega$ to avoid corner singularities.

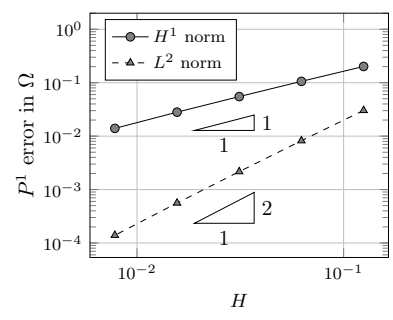

(a)

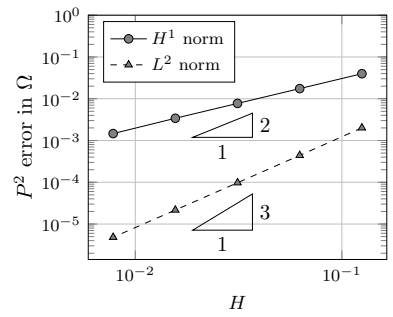

(b)

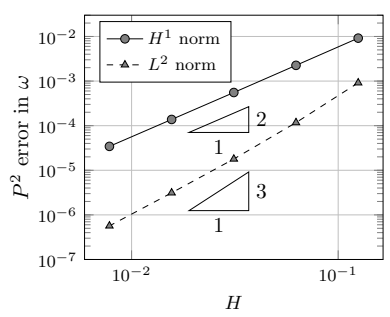

(c)

Fig. 1. Error between $u^{0}$ (solution of (6)) and $u^{H}$ (solution of (8)) in $\Omega$ for (a) $P^{1}$ macro and micro FE spaces and (b) $P^{2}$ macro and micro FE spaces. The setting in (c) is similar to (b) but with $\omega \subset \Omega$. 
Consider now problem (1) with $f=1$, on a $L$-shaped domain centered around $(0,0)$ with width 2 . We impose free Neumann boundary condition on $\{x=$ $0, y \in[-1,0]\}$ and $\{y=0, x \in[0,1]\}$, and homogeneous Dirichlet boundary condition elsewhere.

In Figure 2(a) one can see the reference displacement in comparison to the initial coarse mesh. Using periodic coupling and $\delta=\varepsilon$, we compute the FEHMM solutions for $P^{1}$ macro and micro FE and for $P^{2}$ macro and micro FE; they are shown in Figures 2(b) and 2(c), respectively.

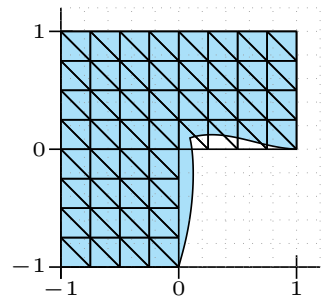

(a)

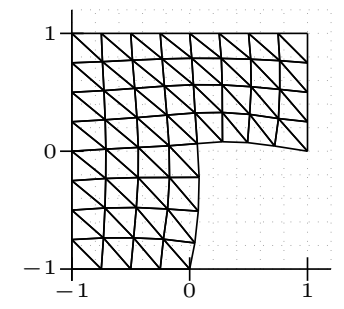

(b)

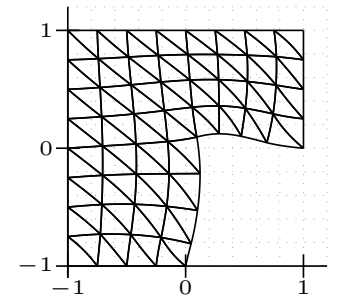

(c)

Fig. 2. (a) Reference solution. Finite element solution $u^{H}$ for $P^{1}$ macro and micro FE (b), and $P^{2}$ macro and micro FE (c).

In Figures 3(a) and 3(b), we plot the $H^{1}$ and $L^{2}$ convergence rates for $P^{1}$ macro and micro FE spaces. The optimal refinement follows the ratio given in Table 1.

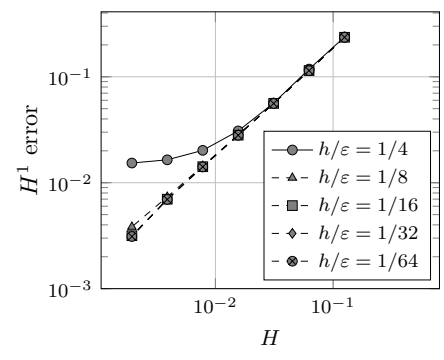

(a)

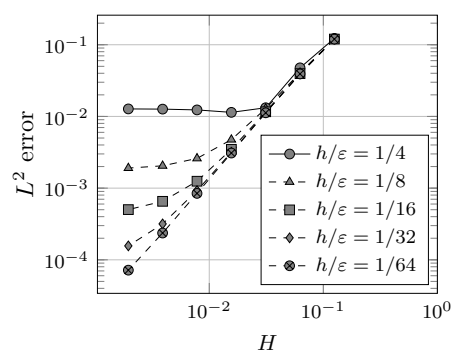

(b)

Fig. 3. $H^{1}$ (a) and $L^{2}$ (b) errors between $u^{0}$ and $u^{H}$ for piecewise macro and micro FE spaces.

We show next the influence of the modeling error on the same problem with sampling domains $K_{\delta}$ with $\delta>\varepsilon$. We take $H=1 / 8,1 / 16,1 / 32$, and $1 / 64$, 
with micro mesh size sufficiently small to eliminate the micro error. We use piecewise $\mathrm{FE}$ for the macro and micro mesh size. The size of the sampling domains $K_{\delta}$ are $\delta=5 / 3 \varepsilon$ and $\delta=1.1 \varepsilon$, and for those values we solve the micro problems (9) with homogeneous Dirichlet boundary conditions. In Figures $4(\mathrm{a})$ and 4(b), we see that the choice of $\delta$ has an important influence in the error. Increasing the size of the sampling domain from $\delta=1.1 \varepsilon$ to $\delta=5 / 3 \varepsilon$ improves the quality of the error, as expected from Theorem 1 . The periodic coupling with $\delta=\varepsilon$ gives the optimal convergence rate since the modeling error is zero, as predicted by Theorem 1 .

Modeling error and random coefficients. The use of artificial boundary conditions for the micro problem (9) leads to a modeling (or resonance) error of size $\mathcal{O}(\varepsilon / \delta)$ for elliptic problems. Such error terms also appear for problems with random stationary fields, where (9) is usually defined in the whole $\mathbb{R}^{d}$ [10]. Truncations using either Dirichlet or periodic boundary conditions can then be used for numerical approximation. In [7], a reduction of this resonance error is obtained by adding a zero-order term the cell problem (9) and using a suitable Richardson extrapolation of the modified cell problem. Such strategies could also be of interest for elastic problems.

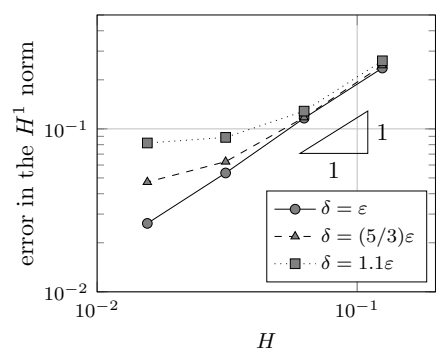

(a)

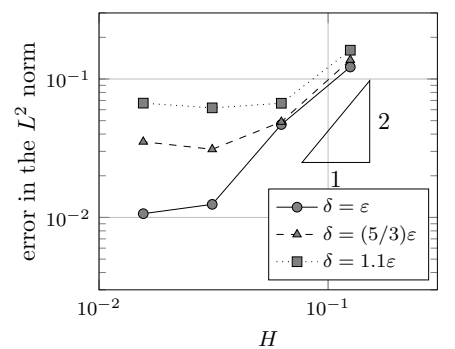

(b)

Fig. 4. $H^{1}$ error (a) and $L^{2}$ error (b) between the homogenized solution and the FE-HMM with Dirichlet coupling for $\delta=5 / 3 \varepsilon$ (dashed) and $\delta=1.1 \varepsilon$ (dotted). The error $\delta=\varepsilon$ (full) is obtained with periodic coupling.

Finally, we study the bound in Lemma 2. We use piecewise FE for the macro problem and compare the exact homogenized tensor with the numerical homogenized tensor. In Figure 5, we show the convergence rate

$$
\left|a_{1111}^{0}-a_{1111}^{0, h}\right|=\left|\sqrt{3}-a_{1111}^{0, h}\right|
$$

for piecewise (full lines) and quadratic (dashed lines) micro FE, and observe the expected rates.

Acknowledgments This work was supported in part by the Swiss National Science Foundation. 


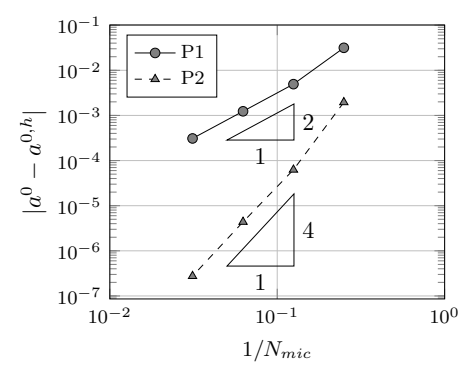

Fig. 5. Convergence rates $\left|a^{0}-a^{0, h}\right|$ with respect to $N_{\text {mic }}^{-1}$ for $P^{1}$ (full) and $P^{2}$ (dashed) micro FE spaces.

\section{References}

1. A. AbDulle, Analysis of a heterogeneous multiscale FEM for problems in elasticity, Math. Models Methods Appl. Sci. 16:4 (2006), 615-635.

2. $\ldots$ The finite element heterogeneous multiscale method: a computational strategy for multiscale PDEs, Multiple scales problems in biomathematics, mechanics, physics and numerics, GAKUTO Internat. Ser. Math. Sci. Appl., vol. 31, Gakkōtosho, Tokyo, 2009, pp. 133-181.

3. _ A priori and a posteriori error analysis for numerical homogenization: a unified framework, Ser. Contemp. Appl. Math. CAM 16 (2011), 280-305.

4. __ Discontinuous Galerkin finite element heterogeneous multiscale method for elliptic problems with multiple scales, Math. Comp. 81:278 (2012), 687-713.

5. G. Allaire, Shape Optimization by the Homogenization Method, Applied Mathematical Sciences, 146, Springer-Verlag New York, 2002.

6. D. Cioranescu And P. Donato, An introduction to homogenization, Oxford Lecture Series in Mathematics and its Applications, vol. 17, Oxford University Press, New York, 1999.

7. A. Gloria And Z. HABIBI, Reduction of the resonance error - part 2: Approximation of correctors, extrapolation, and spectral theory, Preprint, hal-00933234, 2014.

8. F. Murat and L. Tartar, $H$-convergence, Topics in the mathematical modelling of composite materials, Progr. Nonlinear Differential Equations Appl., vol. 31, Birkhäuser Boston, Boston, MA, 1997, pp. 21-43.

9. O. A. Oleinik, A. Shamaev, And G. Yosifian, Mathematical problems in elasticity and homogenization, North-Holland, Amsterdam, 1992.

10. G. C. Papanicolaou And S. R. S. Varadhan, Boundary value problems with rapidly oscillating random coefficients, Random fields, Vol. I, II (Esztergom, 1979), Colloq. Math. Soc. János Bolyai, vol. 27, North-Holland, AmsterdamNew York, 1981, pp. 835-873. 